\title{
Géolinguistique
}

$20 \mid 2020$

Varia

\section{La motivazione lessicale dei nomi d'insetto in tedesco e nelle lingue scandinave}

La motivation lexicale des noms d'insectes dans les langues allemandes et scandinaves

The Lexical Motivation of Insect Names in German and Scandinavian Languages

Claudio Bartoleschi

\section{(2) OpenEdition}

Journals

\section{Edizione digitale}

URL: http://journals.openedition.org/geolinguistique/2032

DOI: 10.4000/geolinguistique.2032

ISSN: 2650-8176

\section{Editore}

UGA Éditions/Université Grenoble Alpes

Edizione cartacea

ISBN: 978-2-37747-246-8

ISSN: 0761-9081

Notizia bibliografica digitale

Claudio Bartoleschi, «La motivazione lessicale dei nomi d'insetto in tedesco e nelle lingue scandinave», Géolinguistique [Online], 20 | 2020, online dal 01 décembre 2020, consultato il 19 janvier 2021. URL: http://journals.openedition.org/geolinguistique/2032 ; DOI: https://doi.org/10.4000/geolinguistique. 2032

Questo documento è stato generato automaticamente il 19 janvier 2021.

Géolinguistique 


\title{
La motivazione lessicale dei nomi d'insetto in tedesco e nelle lingue scandinave
}

\author{
La motivation lexicale des noms d'insectes dans les langues allemandes et \\ scandinaves \\ The Lexical Motivation of Insect Names in German and Scandinavian Languages
}

\author{
Claudio Bartoleschi
}

\section{Introduzione}

1 Questo articolo si propone di classificare e descrivere i diversi modi in cui si formano i nomi di insetto in tedesco e nelle lingue scandinave. I lemmi menzionati sono divisi in quattro grandi categorie a seconda della motivazione lessicale presente nella testa o nel modificatore del composto: categoria «oggetti», «animali», «esseri umani», «creature magiche e sacre». Nella categoria «oggetti», l'insetto è visto come uno strumento usato e controllato da un'entità superiore, generalmente espressa dal modificatore. La categoria «animali» è invece suddivisa in due sottocategorie: «animali di grande taglia», contenente riferimenti ad animali generici o specifici, quali il pollame, gli equini, i bovini, gli ovini, i suini e i cervidi e «designazioni entomologiche», in cui si ritrovano iperonimi legati al mondo dei coleotteri, alle mosche in quanto insetti volanti per antonomasia e ai vermi in quanto loro controparte strisciante. Questa seconda categoria interessa soprattutto la testa del composto, come per esempio nel tedesco Holzwurm e nello svedese trämask «verme del legno», entrambi usati per indicare il tarlo. La categoria «esseri umani» contiene riferimenti generici o specifici relativi a professioni, nazioni e rapporti familiari. Per quanto riguarda la professione, la testa del composto fa riferimento alla persona coinvolta mentre il modificatore specifica quale azione viene svolta o quale oggetto viene usato, come nel danese guldsmed «libellula», letteralmente «orefice», a sua volta divisibile in guld «oro» e smed «fabbro». Le nazionalità vengono invece generalmente usate in modo dispregiativo per indicare 
scarafaggi e altri animali infestanti, come nel tedesco Franzose «francese» o Russe «russo». I nomi familiari vengono utilizzati come vezzegiativi per venerare insetti benevoli, come la coccinella, detta in tedesco anche Himmelsvaterlein «padrino del cielo». La sottocategoria «streghe e megere» rappresenta il punto di congiunzione tra la categoria «esseri umani» e quella delle «creature magiche e sacre». Il riferimento alle streghe può essere tanto nella testa, come nel tedesco Hexenvogel, variante dialettale alsaziana della «libellula», letteralmente «uccello della strega», quanto nel modificatore del composto, come ad esempio nella varianti presenti nel dialetto assiano $\mathrm{e}$ in quello del Palatinato, Wasserhexe, «strega d'acqua». All'ultima categoria appartengono figure sia pagane sia cristiane, come lo svedese trollslända «libellula», letteralmente «fuso del troll», o il danese fandens ridehest «ronzino del diavolo», con riferimento a creature dell'incubo e demoni. Non mancano anche riferimenti a figure positive, quali Dio, la Vergine Maria e altri santi, come nel norreno freyjuhöna «gallina di Freya», da cui poi deriva il norvegese Marihøne "gallina di Maria». Il riferimento a figure antropomorfe, sia umane sia sovranaturali, appare tendenzialmente nel modificatore, che esprime il controllo dell'oggetto o dell'animale presente nella testa.

2 In questa classificazione si è rivelato altresì imprescindibile tener conto dell'importante contributo fornito da Mario Alinei, secondo cui queste categorie iconimiche sarebbero poi analizzabili secondo una stratigrafia archeolinguistica (Alinei, 1997): le designazioni più antiche sarebbero quelle preistoriche di tipo zoomorfico e, in un secondo momento, di tipo antropomorfico, seguite da quelle storiche precristiane e, infine, da quelle cristiane. Le designazioni cristiane, che contano il maggior numero di item a livello zoomorfico in ambito dialettale europeo hanno surclassato un ancor più ampio numero di lemmi precristiani. Solo una piccola parte di questi ultimi è riuscita a mantenersi intatta. Ciò che appare chiaro dai numerosi lemmi analizzati è che l'animale, prima ancora che gli venisse assegnata una designazione, presentava già una sua sacralità, a cui nel corso del tempo sono state poi associate immagini di tipo pagano e cristiano (Alinei, 2002). Molti animali erano infatti originariamente legati a culti totemici e non erano quindi legati a delle entità antropomorfiche superiori. Ad esempio, per l'uomo del paleolitico, il cui sostentamento si basava principalmente sulla caccia, molti animali venivano considerati necessari, e di conseguenza sacri (Alinei, 2002).

3 Per quanto riguarda l'analisi degli item lessicali, mi sono avvalso di diversi dizionari dialettali nelle lingue da me prese in considerazione (lingue scandinave continentali in primis, ossia svedese, norvegese e danese oltre al tedesco, e, in misura minore, l'inglese). In aggiunta, ho anche usufruito di alcune interessanti mappe commentate dell'ALE, come ad esempio quella della coccinella «coccinelle» o della lucciola «ver luisant». A tale proposito, fondamentale è stato il ricorso al sito Wörterbuchnetz, contenente un ampio numero di dizionari dialettali e storici di lingua tedesca e alla pagina web dell'Institutet for språk och folkminnen, istituto svedese di lingua e folclore, contenente diversi dizionari nelle lingue scandinave standard e altrettanti dizionari dialettali. Il lavoro doveva inizialmente coinvolgere solamente le lingue scandinave ma per ottenere dei risultati ottimali era necessario considerare anche ulteriori lingue germaniche, in cui il contributo del cristianesimo si è rivelato più decisivo. In questo modo, è stato altresì reso possibile confermare come molti lemmi inglesi o tedeschi cristiani siano in realtà andati a sostituire precedenti denominazioni pagane 0 , in casi più rari, designazioni legate a culti totemici. Sono partito dall'analisi di quegli insetti di cui era a disposizione maggiore letteratura scientifica, come per esempio la coccinella o 
la farfalla, per poi analizzare insetti più particolari, come la libellula o il tarlo, che si sono poi rivelati altrettanto interessanti. Partendo dal lemma standard presente nelle rispettive lingue scandinave ho poi ricercato diversi sinonimi locali ed eventuali corrispondenze nelle altre lingue germaniche. Quest'analisi ha quindi permesso di notare come le credenze legate ai singoli insetti vadano ben al di là del confine linguistico scandinavo e, in taluni casi, persino al di là dell'area linguistica germanica. La scelta delle categorie lessicali è venuta in seguito alla raccolta dei singoli lemmi: dopo averne infatti raccolto un numero sufficiente, è stato possibile rendersi conto della presenza di alcune categorie ricorrenti alla base della motivazione lessicale degli item considerati e ciò riguarderebbe sia le teste sia i modificatori. Le singole categorie sono accompagnate da alcune tabelle contenenti i vari lemmi, analizzati prevalentemente sulla base della testa del composto. Come già detto in precedenza, per quanto concerne i riferimenti a esseri antropomorfi divini e magici, questi ultimi compaiono nella quasi totalità dei casi nel modificatore. Si ritrova quindi spesso lo stesso lemma all'interno di diverse categorie, in quanto, in taluni casi, è la testa a presentare un dato riferimento alla categoria lessicale menzionata, in altri casi il modificatore.

\section{Oggetti}

\begin{tabular}{|l|l|l|}
\hline Lemma & Traduzione letterale & Designatum \\
\hline trollslända (SV)1 & fuso del troll & libellula \\
\hline nattslända (SV) & fuso della note & libellula \\
\hline sjöspira (SV) & scettro di lago & libellula \\
\hline blindsticka (SV) & ago accecante & libellula \\
\hline djævelens nål (DK) & ago del diavolo & ago del diavolo \\
\hline Teufelsnadel (DE) & ago del diavolo & ago del diavolo \\
\hline hårsnel (SV) & spillo per capelli & libellula \\
\hline Wasserwaage (DE) & bilancia d'acqua & libellula \\
\hline vingalöv (SV) & foglia alata & farfalla \\
\hline
\end{tabular}

4 La motivazione lessicale di termini contenenti riferimenti a oggetti è solitamente di tipo metaforico. La testa del composto in questi casi è solitamente l'oggetto in questione, accompagnato quasi sempre da un modificatore, che specifica a chi appartiene o da chi viene usato l'oggetto. Spesso si tratta di figure sacre tanto pagane quanto cristiane. Un esempio calzante è quello della libellula, facilmente riconoscibile per la sua forma sottile e affusolata, che viene spesso associata a un ago o a uno spillo, come nello svedese standard blindsticka ${ }^{2}$ (spillo accecante), nel dialettale hårsnel (spillo per capelli), nel tedesco Teufelsnadel ${ }^{3}$ (ago del diavolo) o nel corrispondente calco strutturale danese djcevelens nål. Anche in inglese, a livello locale, ritroviamo diverse 
definizioni contenenti l'ago come testa del composto, come in devil's darning/sewing needle (ago per rammendare/cucire). Nello svedese altri oggetti con cui spesso viene associata la libellula sono il fuso, come in trollslända (fuso del troll) e nattslända (fuso della notte), e lo scettro, che ritroviamo nel dialettale sjöspira (scettro del lago). In tedesco la forma aggraziata ed eterea della libellula richiama alla mente il braccio della stadera: ricordiamo, ad esempio, il tedesco dialettale Wasserwaage (bilancia d'acqua) o persino lo standard Libelle, prestito dal latino, che deriva da libella, diminutivo di libra, ovvero una piccola bilancia. Un altro esempio su base metaforica è quello della farfalla, le cui ali possono ricordare addirittura delle foglie, come nella regione svedese del Blekinge, dove viene chiamata vingalöv (foglia alata) (Nilsson, 2008).

\section{Animali}

\subsection{Animali di grande taglia}

5 Il legame che intercorre tra insetti e animali più grandi non è sempre facile da motivare anche se non sono mancati nel corso del tempo diversi tentativi di dirimere la questione. Solo in taluni casi sembra prevalere una motivazione lessicale di tipo metaforico basata sulla somiglianza tra l'insetto e l'animale. Come affermato da Mario Alinei, in molti casi c'entrano poco o nulla la forma o le caratteristiche fisiche. La discriminante sembra essere piuttosto la funzione magica che gli animali hanno nelle fiabe, dove ricoprono il ruolo di assistenti dell'eroe, mettendolo in condizione di superare le proprie difficoltà e raggiungere la meta prefissata. Allo stesso modo, in molte rime infantili, gli insetti venivano invocati in cerca di protezione e sostegno da parte del bambino, che voleva vedere realizzati i propri desideri. Ricorrendo ai mezzi a loro disposizione, i bambini tendevano quindi a paragonare gli insetti agli animali con cui avevano maggiore dimestichezza e con cui, probabilmente, avevano anche instaurato un qualche rapporto di tipo affettivo. Identificando l'insetto con l'animale, il bambino mostrava il proprio apprezzamento e attaccamento emotivo nella speranza di veder esaudito il proprio desiderio. L'idea era quindi quella di descrivere l'ignoto attraverso ciò che invece era noto. In aggiunta a quanto detto finora, Mario Alinei fa giustamente notare che potremmo quasi considerare il mondo degli insetti la versione miniaturizzata del nostro, dove gli insetti svolgono la stessa funzione svolta dagli uomini e dagli animali più grandi, solo in scala molto più ridotta (Alinei \& Barros Ferreira, 1990).

\section{Animali di grande taglia: denominazioni generiche}

\begin{tabular}{|l|l|l|}
\hline Lemma & Traduzione letterale & Designatum \\
\hline Gottestierchen (DE) & animaletto di Dio & coccinella \\
\hline Sommertierchen (DE) & bestiola estiva & farfalla/coccinela \\
\hline Buttervogel (DE) & uccello del burro & farfalla \\
\hline Hexenvogel (DE) & uccello della strega & libellula \\
\hline
\end{tabular}




\begin{tabular}{|l|l|l|}
\hline Johannisvögelchen (DE) & uccellino di San Giovanni & lucciola \\
\hline Maivogel (DE) & uccello di maggio & farfalla/coccinela \\
\hline Sommervöglein (DE) & uccello estivo & coccinella \\
\hline sommerfugl (NO, DK) & uccello estivo & farfalle \\
\hline sommarfågel (SV) & uccello estivo & farfalle \\
\hline
\end{tabular}

6 Nelle lingue germaniche la testa del composto può contenere un riferimento piuttosto generico e banale, portando il parlante a focalizzare l'attenzione sul modificatore, che veicola pertanto l'informazione più importante. Prendiamo ad esempio due lemmi tedeschi usati per indicare la coccinella e la farfalla: Gottestierchen "animaletto di Dio» ${ }^{4}$ e Sommertierchen «animaletto estivo» 5 . Sia nel primo sia nel secondo caso l'attenzione è rivolta al modificatore più che al fatto che si tratti effettivamente di un animale di piccole dimensioni. Molti insetti volanti vengono poi definiti «uccelli». Gli uccelli infatti fungono da tramite tra la terra e il cielo, i vivi e i morti e, in diverse tradizioni popolari, simboleggiano l'anima umana. Non sorprende quindi che si pensi che alcuni insetti volanti, come la coccinella e la farfalla, uniscano il mondo terreno all'aldilà. C'è inoltre da puntualizzare che nelle lingue germaniche, fatta eccezione per l'inglese, il termine stesso per uccello contiene nella propria radice l'idea del volo e quindi esso stesso potrebbe essere considerato un termine generico da usare per designare qualsiasi animale in grado di volare, inclusi gli insetti. Anche qui, come negli esempi menzionati sopra, il modificatore fa riferimento alla stagione o al mese di apparizione dell'insetto, come nello scandinavo sommarfágel/sommerfugl «uccello estivo» ${ }^{6}$ o nel tedesco Maivogel «uccello di maggio», entrambi indicanti la farfalla, o come nel Tedesco Sommervöglein "uccellino estivo» ${ }^{8}$, che indica invece la coccinella. Altri termini quali Hexenvogel «uccello della strega»", per indicare la libellula, e Johannisvögelchen "uccellino di Giovanni $»^{10}$, per indicare invece la lucciola, hanno una figura sacra o magica nel modificatore. Stesso discorso vale anche per l'inglese ladybird «uccello della signora», dove una figura sacra sia essa pagana o cristiana controlla un animale volante, nella fattispecie la coccinella. Persino il termine Buttervogel «uccello del burro» ${ }^{11}$ fa implicitamente riferimento alle streghe ma avremo modo di approfondire la questione successivamente.

\section{Pollame}

\begin{tabular}{|l|l|l|}
\hline Lemma & Traduzione letterale & Designatum \\
\hline Gullhöna (SV) & gallina d'oro & coccinella \\
\hline Mariehøne (NO, NN, DK) & gallina di Maria & coccinella, farfalla \\
\hline Mariehøns (NO, NN) & pollo di Maria & coccinella \\
\hline Akerhøne (NO, NN) & gallo del campo & coccinella \\
\hline Agerhøne (DK) & gallo del campo & coccinella \\
\hline
\end{tabular}




\begin{tabular}{|l|l|l|}
\hline Fårekylling (DK) & pollo del pericolo & grillo \\
\hline Paradishane (DK) & gallo del paradiso & coccinella \\
\hline Vorherreshane (DK) & gallo del Signore & coccinella \\
\hline Liebergottshahn (DE) & gallo del Caro Signore & coccinella \\
\hline Springhahn (DE) & gallo saltellante & cavalletta \\
\hline
\end{tabular}

7 Tra i nomi di insetti più comuni ci sono sicuramente quelli che presentano un riferimento al gallo o alla gallina.

In una delle fiabe di Esopo la gallina aveva la capacità di deporre uova d'oro (Esopo, 2016) e questo nesso tra il pollame e la ricchezza risulta essere un tema ricorrente in diverse tradizioni popolari (Alinei \& Barros Ferreira, 1990). Allo stesso modo, le coccinelle, e in misura minore, le farfalle venivano invocate nelle rime infantili in quanto portatrici di fortuna, felicità e ricchezza, come dimostrato in questa rima infantile svedese:

Gullhöna, gullko!

Flyg öster, flyg vester,

Dit du flyger der bor din älskade!

Gallina d'oro, mucca d'oro (coccinella)

vola a est, vola a ovest

là dove volerai vive il tuo amato!

Questa versione della rima infantile è comune nel sud della Svezia e ha come protagonista la gallina d'oro gullhöna, portatrice di prosperità e benessere economico come viene riportato nella tradizione popolare (Hendriks, 2017). Inoltre, viene mostrato anche come tra i vari poteri magici attribuiti alla coccinella, ci sia quello di indicare alle spose da che direzione arriverà il futuro sposo. Sull'isola danese di Funen invece gli abitanti erano soliti invocare la coccinella sperando di far migliorare il tempo:

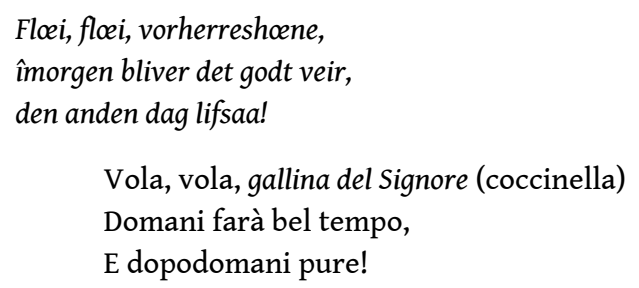

In norvegese e in danese standard la coccinella è spesso anche associata alla gallina della Vergine Maria, come dimostrato dai termini mariehøne e marihøns ${ }^{12}$. In questo caso la coccinella funge da intermediario della Vergine Maria, esaudendo i desideri del richiedente. Appaiono poi esclusivamente in danese e norvegese designazioni della coccinella quali agerhøne e paradishane, letteralmente "gallo del campo» e "gallo del paradiso", che vengono usati normalmente come significanti di altri tipi di pollame, nella fattispecie due distinte razze di fagiano (Alinei \& Barros Ferreira, 1990). Nel tedesco dialettale Springhahn "grillo» (letteralmente «gallo saltellante») e nel danese standard fårekylling «cavalletta» (letteralmente "pollo pericoloso») non si rivela alcuna motivazione di tipo magico-religioso: Får-deriva dall'Antico Nordico fara «pericolo» e 
dipende probabilmente dal fatto che i grilli infestino i campi e il raccolto mentre il riferimento al pollo potrebbe dipendere dal suono prodotto dal pollame, che viene ritenuto simile a quello prodotto dalla cavalletta. Anche Springhahn ha una motivazione simile: le cavallette producono suoni che possono vagamente ricordare quelli del pollame e inoltre saltano, il che motiverebbe il modificatore Spring-(springen «saltare») $)^{13}$. In entrambi questi casi, gli insetti sembrerebbero copiare alcune caratteristiche tipiche di animali più grandi.

\section{Equini}

\begin{tabular}{|l|l|l|}
\hline Designazione & Traduzione letterale & Designatum \\
\hline brudehest (NO) & cavallo della sposa & coccinella \\
\hline Liebgottrösslein (DE) & destriero del Buon Signore & coccinella \\
\hline Teufelspferd (DE) & cavallo del diavolo & libellula \\
\hline
\end{tabular}

11 La coccinella viene spesso associata anche al cavallo, come dimostrano lemmi quali il norvegese brudehest «cavallo della sposa» (Hendriks, 2017) e il tedesco Liebgottrösslein «destriero del buon Signore». In brudehest, alla coccinella viene richiesto di mostrare la direzione da cui arriverà il futuro sposo, a convalida della rima infantile incontrata in precedenza. Simili sono anche lo svedese brudevisare e visbrud (letteralmente «che mostra alla sposa») (Nilsson, 2008). Anche la libellula è spesso vista come un cavallo, come nel tedesco Teufelspferd «cavallo del diavolo» ${ }^{14}$. Anche in questo caso, l'insetto viene percepito come un animale o un oggetto controllato da forze superiori e, nel caso specifico, interpretato come epifania del male, ovvero come una creatura legata al mondo dell'incubo.

\section{Ovini}

\begin{tabular}{|l|l|l|}
\hline Designazione & Traduzione letterale & Designatum \\
\hline Geting (SV) & capra + suffisso '-ing' & Vespa \\
\hline Gedehams (DK) & vespa della capra & Vespa \\
\hline Hornisse (DE) & creatura con le corna & Vespa \\
\hline Himmelsschäfchen (DE) & pecorella del cielo & coccinella \\
\hline Rebbock (DE) & caprone della vite & cervo volante \\
\hline Heubock (DE) & caprone del fieno & Cavalletta \\
\hline Haferbock (DE) & caprone dell'avena & Cavalletta \\
\hline Skinnbagge (SV) & caprone di pelle & Acaro \\
\hline
\end{tabular}




\begin{tabular}{|l|l|l|}
\hline Skalbagge (SV) & caprone con il guscio & coleottero \\
\hline Hjortbagge (SV) & caprone cervo & cervo volante \\
\hline Honnbagge (SV) & caprone con le corna & cervo volante \\
\hline Torbagge (SV) & caprone dello sterco & scarabeo stercorario \\
\hline Majbagge (SV) & caprone di maggio & maggiolino, coccinella \\
\hline
\end{tabular}

12 Molti di questi nomi d'insetto non presentano alcuna motivazione lessicale di tipo magico-religioso ma si basano solitamente su una relazione di tipo metaforico e/o metonimico. È, ad esempio, di base metaforica la motivazione lessicale dello svedese geting «vespa» (letteralmente "capra» + suffisso derivazionale in -ing). Anche qui si ricorre al noto per descrivere l'ignoto: le antenne della vespa richiamano infatti alla memoria le corna di un montone. Quindi, l'insetto in questo caso si limita esclusivamente a riprodurre le caratteristiche fisiche di un animale più grande e con cui si ha maggiore dimestichezza ${ }^{15}$. Lo stesso vale anche per il danese gedehams «vespa» dove il modificatore del composto rimanda sempre alla capra (danese ged) mentre l'origine della testa rimane in questo caso sconosciuta ${ }^{16}$. Anche il composto inglese hornet e il Tedesco Hornisse ${ }^{17}$, entrambi usati per designare il calabrone, usano le antenne dell'animale come elemento distintivo. Il termine per caprone, Bock ricorre in parecchi nomi dialettali tedeschi usati per descrivere diversi insetti, come Rebbock «caprone della vite», Heubock «caprone del fieno» ${ }^{18}$, Haferbock «caprone dell'avena» (Bächthold-Stäubli \& Hoffmann-Kreyer, 1986). Heubock, in particolar modo, viene usato come significante della cavalletta, insieme al ben più noto Heuschrecke «saltatore del fieno» (Schrecken in antico tedesco significava per l'appunto «saltare»), interpretabile anche come «spavento del fieno» (ted. Schreck «spavento»). Secondo il folklore germanico, la cavalletta era considerata uno spirito dei campi (ted. Feldgeist) o demone del grano (ted. Korndämon), conosciuto con il nome di Haferbock «montone dell'avena». Questi spiriti erano inizialmente considerati positivi e venivano associati ad animali da allevamento quale il montone. Ai tempi delle civiltà agricole, erano considerati divinità della vegetazione che abitavano e proteggevano i campi di grano ma, con il passare del tempo, si sono tramutati in creature malefiche utilizzate come monito per scoraggiare i bambini dall'allontanarsi nell'erba alta e distruggere il raccolto (Mannhardt, 2018). Anche lo svedese bagge era originariamente usato per indicare il montone mentre il suo composto skalbagge (letteralmente "caprone con il guscio») veniva riservato come termine generico per indicare i coleotteri. In tempi più recenti, anche il termine base bagge ha iniziato a essere utilizzato come significante per indicare i coleotteri e, in qualità di testa del composto, si trova in molti nomi d'insetto: lo scarabeo è ad esempio chiamato hjortbagge "coleottero cervo» o hornbagge "coleottero con le corna»; il maggiolino majbagge "coleottero di maggio»; l'acaro skinnbagge "coleottero della pelle»; lo scarabeo stercorario torbagge. In quest'ultimo caso, il modificatore tor- è imparentato con il lessema antico inglese tord «sudiciume», che è poi proseguito nell'inglese contemporaneo con la parola turd «escremento» ${ }^{19}$. Per ultima, menzioniamo anche la pecora, che compare nel termine tedesco Himmelsschäfchen "pecorella del cielo». Quest'ultima può far riferimento a due diversi designata legati tra loro: la coccinella (questo è il significato primario nella regione della Renania) e un fiore, la primula veris 
(questo significato prevale invece nella regione del Palatinato ${ }^{20}$ ). Questo tema verrà approfondito nel paragrafo su Freya e la Vergine Maria.

Suini

\begin{tabular}{|l|l|l|}
\hline Lemma & Traduzione letterale & Designatum \\
\hline Gullgris (SV) & maiale d'oro & coccinella \\
\hline Gullsugga (SV) & scrofa d'oro & coccinella \\
\hline
\end{tabular}

13 Non è ben chiaro cosa porti a scegliere un animale anziché un altro. Per quanto ci riguarda, tutti gli animali nominati finora, o che nomineremo in seguito, facevano parte della quotidianità dell'individuo e, in gran parte dei casi, rappresentavano un'indiscutibile fonte di sostentamento. A onor del vero, i nomi di insetto contenenti riferimenti ai suini scarseggiano nelle lingue germaniche. In svedese ne abbiamo due ed entrambi si riferiscono alla coccinella: gullgris «maiale d'oro» e gullsugga «scrofa d'oro». Il termine gullgris è usato in svedese anche per indicare un bambino particolarmente viziato, quindi la coccinella doveva essere adorata, onorata e, in un certo qual modo, viziata, affinché l'uomo potesse veder esaudito il proprio desiderio. Anche qui appare chiaro, come abbiamo avuto modo di precisare in precedenza, quanto l'enfasi sia più sul modificatore che sulla testa: l'insetto veniva invocato per ottenere beni materiali, da qui il riferimento all'oro. Poi, che si trattasse di un maiale, di una mucca o di un cavallo, nella maggior parte dei casi non rappresentava poi una differenza così rilevante. Erano, dopo tutto, tutti animali allevati e controllati dall'uomo. Probabilmente in tal modo si pensava di poter soggiogare ed esorcizzare anche quegli elementi che sfuggivano al controllo dell'uomo, come per esempio gli insetti, tramite il riferimento al noto, al conosciuto, al familiare. Tornando ai suini, sono più comuni i riferimenti al maiale nelle lingue romanze, come nell'italiano «porcello di Sant'Antonio» o nel corrispettivo francese cochon de saint Antoine (Alinei \& Barros Ferreira, 1990), tanto che, a livello iconografico, spesso Sant'Antonio abate è raffigurato con un maiale ai suoi piedi. Si crede infatti che il maiale sia stato salvato e guarito dal santo e che rappresenti simbolicamente i poveri e gli oppressi. Anche in questo caso, l'animale ha valore di intermediario tra una figura sacra e l'uomo, ricoprendo quindi il ruolo di intermediario e di messaggero.

\section{Bovini}

\begin{tabular}{|l|l|l|}
\hline Lemma & Traduzione letterale & Designatum \\
\hline gullko (SV) & mucca d'oro & coccinella \\
\hline Motschekiebchen (DE) & piccolo mucca muggente & coccinella \\
\hline Muhkälbchen (DE) & vitellino muggente & coccinella \\
\hline Motschekälbchen (DE) & vitellino muggente & coccinella \\
\hline
\end{tabular}




\begin{tabular}{|l|l|l|}
\hline Sommerkälbchen (DE) & vitellino estivo & coccinella \\
\hline
\end{tabular}

14 Tra i principali animali allevati dall'uomo vale la pena menzionare anche i bovini. Abbiamo già incontrato nella rima infantile svedese menzionata prima lo svedese gullko «mucca d'oro», invocata per ottenere ricchezza e beni materiali. Altri nomi della coccinella sono il tedesco Motschekälbchen e Muhkälbchen «piccola mucca che muggisce» entrambi contenenti l'onomatopeico motsche-/muh-, indicanti il muggito prodotto dal bovino ${ }^{21}$.

\section{Cervo}

\begin{tabular}{|l|l|l|}
\hline Lemma & Traduzione letterale & Designatum \\
\hline Eghjort (DK) & cervo della quercia & cervo volante \\
\hline Eikehjort (NO, NN) & cervo della quercia & cervo volante \\
\hline flygande hjort (SV) & cervo volante & cervo volante \\
\hline Fliegender Hirsch (DE) & cervo volante & cervo volante \\
\hline Hirschmann (DE) & uomo cervo & cervo volante \\
\hline Hirschochse (DE) & bue cervo & cervo volante \\
\hline
\end{tabular}

15 Anche i nomi di insetto aventi il cervo come testa del composto sono principalmente di tipo metaforico. Lo stesso "cervo volante» ne è una chiara dimostrazione, visto che le corna dell'animale ricordano vagamente proprio quelle del cervo. I nomi del cervo volante nelle lingue germaniche hanno come modificatore del composto o un termine che indichi il fatto che l'animale voli, come nelle lingue romanze, o come nel tedesco Fliegender Hirsch e nello svedese flygande hjort, entrambi traducibili letteralmente proprio con «cervo volante», oppure fanno riferimento all'habitat dell'insetto, come nel danese e nel norvegese eghjort e ekhjort "cervo della quercia» ${ }^{22}$. In svedese il termine più usato è ekoxe "bue della quercia», anche quest' animale è infatti munito di corna, proprio come il cervo. Sono presenti poi anche i due composti coordinativi tedeschi Hirschmann «uomo cervo» ${ }^{23}$ e Hirschochse «bue cervo».

\subsection{Generalizzazioni a livello entomologico}

\section{Coleottero}

\begin{tabular}{|l|l|l|}
\hline Lemma & Traduzione letterale & Designatum \\
\hline Marienkäfer (DE) & coleottero di Maria & Coccinella \\
\hline Herrgottskäfer (DE) & coleottero del Signore & coccinella \\
\hline Muttergotteskäfer (DE) & coleottero della madre di Dio & coccinella \\
\hline
\end{tabular}




\begin{tabular}{|c|c|c|}
\hline Leuchtkäfer (DE) & coleottero Luminoso & lucciola \\
\hline Johanniskäfer (DE) & coleottero di San Giovanni & lucciola \\
\hline Frauenkäferlein (DE) & piccolo coleottero della signora & coccinella \\
\hline Glückskäfer (DE) & coleottero della gioia/fortuna & coccinella \\
\hline Maikäfer (DE) & coleottero di maggio & maggiolino/coccinella \\
\hline Junikäfer (DE) & coleottero di giugno & coccinella \\
\hline Sommerkäfer (DE) & coleottero estivo & coccinella \\
\hline Sonnwendkäfer (DE) & coleottero del solstizio & coccinella, lucciola \\
\hline Goldkäfer (DE) & coleottero d'oro & lucciola \\
\hline Nagekäfer (DE) & coleottero che mordicchia & tarlo \\
\hline Bohrkäfer (DE) & coleottero perforante & tarlo \\
\hline Klopfkäfer (DE) & coleottero che bussa & tarlo \\
\hline Lysbille (NO, DK) & coleottero della luce & lucciola \\
\hline Ljosbille (NN) & coleottero della luce & lucciola \\
\hline Borebille (NO, NN, DK) & coleottero perforante & tarlo \\
\hline Hjortebille (NO, NN) & coleottero cervo & cervo volante \\
\hline Pilletrillebille (NO) & coleottero stercorario & cervo volante \\
\hline Skinnbagge (SV) & coleottero di pelle & acaro \\
\hline Skalbagge (SV) & coleottero con il guscio & coleottero \\
\hline Hjortbagge (sv) & coleottero cervo & cervo volante \\
\hline Honnbagge (SV) & coleottero con le corna & cervo volante \\
\hline Torbagge (SV) & coleottero dello sterco & scarabeo stercorario \\
\hline Majbagge (SV) & coleottero di maggio & maggiolino \\
\hline
\end{tabular}

16 Come detto già in precedenza, lo svedese bagge può essere utilizzato per indicare sia il montone sia come termine generico per i coleotteri. Anche le altre lingue germaniche hanno un termine generico indicante i coleotteri, a cui, poi nella formazione dei composti, viene aggiunto un determinato modificatore, che rende il termine, inizialmente generico, specifico di un tipo d'insetti: il norvegese e il danese usano bille

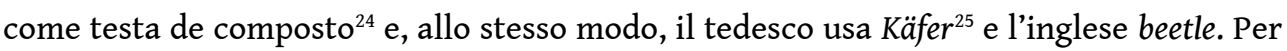


quanto riguarda i modificatori, il tedesco ricorre spesso a riferimenti cristiani: Marienkäfer «coleottero di Maria», Muttergotteskäfer "coleottero della madre di Dio», Herrgottskäfer "coleottero di Dio» ${ }^{26}$ e Johanniskäfer "coleottero di san Giovanni»" ${ }^{27}$. Il tedesco è infatti, insieme all'inglese, la lingua germanica dove l'influsso del cristianesimo è stato più rilevante. Il riferimento alla vergine Maria, e in misura minore, agli altri santi, è solitamente legato al fatto che la coccinella venga vista come la versione cristianizzata di dee pagane dell'antico pantheon germanico quali Freya e Frigg (Hendriks, 2017). Oltre a essere invocata per ottenere ricchezze, per incontrare il proprio futuro sposo o per scongiurare la pioggia, la coccinella è anche vista come portatrice di felicità e fortuna, come dimostrato nel tedesco Glückskäfer «coleottero della felicità/della fortuna».

In altri casi, è invece il mese o la stagione in cui appare l'insetto, a essere indicato nel modificatore. Sempre per la coccinella abbiamo infatti il tedesco Junikäfer «coleottero di giugno»; il tedesco Maikäfer e lo svedese majbagge «coleottero di maggio» e ancora il tedesco Sommerkäfer "coleottero estivo» e Sonnwendkäfer "coleottero del solstizio d'estate». Anche la lucciola è un insetto estivo e appare solitamente a inizio estate: infatti viene chiamata anch'essa Sommerkäfer o Sonnwendkäfer, proprio come la coccinella (Alinei \& Barros Ferreira, 1990). Anche Johanniskäfer ha a che fare con il momento in cui l'insetto fa la sua comparsa: infatti il giorno di San Giovanni cade approssimativamente negli stessi giorni del solstizio. Quindi può essere giustamente considerato una versione cristianizzata dello stesso periodo.

Il modificatore, inoltre, oltre a poter far riferimento a una figura sacra e al mese o stagione in cui appare l'insetto, può far riferimento all'azione compiuta dall'insetto stesso: il tedesco Nagekäfer "coleottero che mordicchia», Bohrkäfer "coleottero perforante», Klopfkäfer "coleottero che bussa» ${ }^{28}$ e il norvegese/danese borebille «coleottero che perfora» entrambi usati per il tarlo, sono degli esempi perfetti di questo tipo di composto.

Tornando ad altri nomi della lucciola, l'elemento distintivo di questo insetto è senza dubbio quello di emettere luce ed è infatti questo aspetto a essere sottolineato molte volte nel modificatore, come mostratoci nel tedesco Leuchtkäfer e nel norvegese/danese lysbille «coleottero luminoso». Per questo motivo viene altresì associato a elementi preziosi e luccicanti, da qui il tedesco Goldkäfer «coleottero dorato».

Nel paragrafo sul cervo, abbiamo visto come anche il cervo, come in genere tutti gli animali presenti nei nomi di insetto, sia di solito la testa del composto. Non è sempre così: infatti il cervo può essere anche il modificatore del composto, specie quando accompagna termini più generici come "coleottero»: ecco spiegati termini quali il tedesco Hirschkäfer, lo svedese hjortbagge e il norvegese hjortbille, «coleottero cervo».

\section{Mosca}

\begin{tabular}{|l|l|l|}
\hline Lemma & Designazione letterale & Designatum \\
\hline Ildflue (NO, DK) & insetto volante di fuoco & lucciola \\
\hline Eldfluga (SV) & insetto volante di fuoco & lucciola \\
\hline Lysfluga (SV) & insetto volante della luce & lucciola \\
\hline
\end{tabular}


Johannisfliege (DE) insetto volante di San Giovanni lucciola

21 Nonostante nelle lingue romanze il riferimento alla mosca possa far pensare a un insetto specifico, nelle lingue germaniche, il termine per mosca ha in sé la radice germanica contenente l'idea del volo e quindi può riferirsi a qualsiasi insetto in grado di volare (Barros Ferreira, 1997). Essendo la mosca l'insetto volante per antonomasia è probabile che il termine, inizialmente generico, abbia subito un successivo restringimento semantico. Quindi, come il termine per uccello conteneva in sé l'idea di volo e poteva riferirsi a qualsiasi animale volante (de. Vogel, sv. fågel, no/dk fugl), anche «la mosca» (ing. fly, de. Fliege e sv. fluga) poteva riferirsi a qualsiasi insetto in grado di volare. Viene infatti usato come testa del modificatore in alcuni nomi di insetto come nello svedese lysfluga «mosca/insetto volante della luce» ed eldfluga «mosca/insetto volante del fuoco», entrambi lemmi che indicano la lucciola. In tedesco la testa Fliege viene accompagnata dal modificatore Johannis, San Giovanni, che come detto in precedenza indica il periodo di comparsa dell'insetto ${ }^{29}$.

\section{Verme}

\begin{tabular}{|l|l|l|}
\hline Lemma & Traduzione letterale & Designatum \\
\hline Træorm (DK) & verme del legno & tarlo \\
\hline Trämask (SV) & verme del legno & tarlo \\
\hline Tremark (NO) & verme del legno & tarlo \\
\hline Holzwurm (DE) & verme del legno & tarlo \\
\hline Lysmask (SV) & verme della luce & lucciola \\
\hline Glismask (SV) & verme della luce & lucciola \\
\hline Glühwürmchen (DE) & verme luccicante & lucciola \\
\hline Joahnniswurm (DE) & verme di San Giovanni & lucciola \\
\hline Sonnenwürmchen (DE) & vermicello del sole & coccinella \\
\hline Brutwürmchen (DE) & vermicello della sposa & coccinella \\
\hline Himmelswurm (DE) & verme del cielo & coccinella \\
\hline Marienwürmchen (DE) & verme di Maria & coccinella \\
\hline
\end{tabular}

«Verme» nei composti delle lingue germaniche è usato qui come iperonimo per designare non solo qualsiasi insetto strisciante ma, spesso e volentieri, anche un qualsiasi tipo di insetto. Il termine si riferiva originariamente sia ai vermi sia ai serpenti essendo entrambi animali striscianti. Le diverse lingue germaniche sono andate poi man mano specializzandosi preferendo l'uno o l'altro significato ${ }^{30}$. L'inglese 
e il tedesco usano rispettivamente worm e Wurm per indicare il verme. Allo stesso modo il danese per influsso del basso tedesco, e a differenza delle altre lingue scandinave, usa orm per verme e slange per serpente. Invece, lo svedese e il norvegese usano entrambi orm per indicare il serpente e lo svedese mask o il norvegese mark per il verme. Quest'ultimo deriva dalla radice dell'antico germanico mađ «rosicchiare» e si ritrova anche in altri lemmi, quali l'inglese moth e il tedesco Motte, «falena» ${ }^{31}$. Nei diversi composti, avremo quindi o l'uno o l'altro lemma come testa accompagnato da un modificatore, tendenzialmente uguale o molto simile. Il tarlo, per esempio, viene chiamato "verme del legno» (inglese woodworm, tedesco Holzwurm, danese trcorm, norvegese tremark e svedese trämask):

\begin{tabular}{|l|l|l|l|l|}
\hline ing. & wood & & worm \\
\cline { 1 - 1 } ted. & Holz & & Wurm & \multirow{2}{*}{ verme } \\
\cline { 1 - 2 } dan. & tree & legno & orm & \\
\cline { 1 - 1 } norv. / sved. & tre / trä & & mark / mask & \\
\hline
\end{tabular}

La stessa cosa vale anche per la lucciola, come nel tedesco Glühwürmchen (vermetto luccicante) e nello svedese glismask/lysmask (verme luccicante/luminoso). Altri lemmi contenenti «verme» come testa del composto sono alcuni nomi della coccinella: Sonnenwürmchen «vermetto del sole», Brautwürmchen «vermetto della sposa» (la aiuta a trovare marito), Himmelswurm «vermetto del cielo» e Marienwürmchen «vermetto di Maria».

\section{Esseri umani}

\section{Generalizzazioni}

\begin{tabular}{|l|l|l|}
\hline Lemma & Traduzione letterale & Designatum \\
\hline Nyckelpiga (SV) & serva della chiave & coccinella \\
\hline Kärnanpiga (SV) & serva della zangola & coccinella \\
\hline Jessvallpika (SV) & pastorella di Gesù & coccinella \\
\hline Fårpiga (SV) & serva della pecora & coccinella \\
\hline Getflicka (SV) & ragazza della capra & coccinella \\
\hline Getpojke (SV) & ragazzo della capra & coccinella \\
\hline Sonnenkindchen (DE) & bambinello del sole & coccinella \\
\hline Wasserjungfrau (DE) & vergine dell'acqua & libellula \\
\hline vandnymf (DK) & ninfa dell' acqua & libellula \\
\hline
\end{tabular}




\begin{tabular}{|l|l|l|}
\hline vannnymfe (NO) & ninfa dell'acqua & libellula \\
\hline Guldbrud (SV) & sposa d'oro & coccinella \\
\hline visbrud (NO) & sposa che mostra [...] & coccinella \\
\hline Gullkonge (NO) & re d'oro & coccinella \\
\hline
\end{tabular}
questi prevalgono senza alcun dubbio nella testa dei composti, visto che gli insetti hanno generalmente funzione di messaggeri o servi di entità superiori. Il tedesco Wasserjungfrau «vergine delle acque» indica la libellula o, per essere più precisi, una sottospecie conosciuta come damigella. Il termine italiano, come avviene d'altronde con l'inglese damsel (in inglese questa specie di libellule si chiama damselfly), è imparentato con il francese demoiselle e indica, proprio come in tedesco, una donna di giovane età non sposata e senza alcuna precedente esperienza sessuale. Dato che questo riferimento allude chiaramente all'elegante forma affusolata della libellula, il legame tra insetto ed essere umano menzionato è di tipo metaforico. In senso lato, il termine Wasserjungfrau, nel tedesco svizzero, è andato poi a indicare la canna del giunco, che presenta lo stesso aspetto affusolato della libellula ${ }^{32}$. L'aspetto elegante dell'insetto è presente anche nelle lingue scandinave con lo svedese flickslända «fuso della ragazza» e jungfruslända "fuso della vergine» come anche nel danese vandnymfe e nel norvegese vannymfe «ninfa delle acque» ${ }^{33}$. Nel caso del norvegese e del danese il rapporto metaforico è basato sul legame con le ninfe, giovani divinità femminili che vivono nei pressi degli specchi d'acqua mentre nei lemmi svedesi il fuso richiama la forma dell'insetto.

I desideri che il richiedente vuole vedere esauditi sono generalmente gli stessi che abbiamo menzionato in precedenza, tra cui il desiderio di arricchirsi. Ciò giustificherebbe la presenza dell'oro come modificatore di diversi composti, quali il norvegese gullkonge «re d'oro» e lo svedese guldbrud «sposa d'oro». In svedese una guldbrud è anche una sposa nel giorno del suo matrimonio e potrebbe essere quindi visto come un buon auspicio per tutte coloro che vogliono o sono in procinto di sposarsi ${ }^{34}$. In altre definizioni, alla coccinella viene richiesto di far palesare il futuro sposo o di indicarne la direzione di provenienza, come nello svedese brudvisare e visbrud. La sposa compare spesso come modificatore del composto accompagnando la testa (un animale o un insetto): un esempio è il tedesco Brautwurm «verme della sposa» o il norvegese brudehest «cavallo della sposa». L'intervento della coccinella è spesso richiesto anche per portare il bel tempo, come nel tedesco Sonnenkindchen «bambinello del sole».

\section{Professioni}

\begin{tabular}{|l|l|l|}
\hline Lemma & Traduzione letterale & Designatum \\
\hline Schuhmacher (DE) & calzolaio & $\begin{array}{l}\text { coccinella, farfalla, libellula, ragno, } \\
\text { cervo volante, tarlo, cavalletta }\end{array}$ \\
\hline
\end{tabular}




\begin{tabular}{|l|l|l|}
\hline Goldschmied (DE) & orefice & coccinella \\
\hline Guldsmed (DK) & orefice & libellula \\
\hline Gullsmed (SV, NO) & orefice & coccinella \\
\hline Getare (SV) & pastore & coccinella \\
\hline Geterska (SV) & pastorella & coccinella \\
\hline
\end{tabular}

La figura del pastore si ritrova in diversi nomi svedesi della coccinella, quali fårpiga, (Jess)vallpika, getflicka, getpojke, getare e geterska. Il pastore è legato al suo gregge da un vincolo speciale, quasi sacro tanto che l'immagine del pastore che guida il suo gregge ricorre spesso anche nel mondo cristiano. Secondo il folclore locale, la coccinella protegge pecore e capre e rivolgere a essa preghiere e devozione assicurerebbe la sicurezza del gregge (Alinei \& Barros Ferreira, 1990). Spesso alla coccinella veniva fatta una richiesta ben specifica all'interno delle rime infantili. La coccinella veniva invocata al fine di portare ricchezza al richiedente: lemmi quali lo svedese gullsmed e il tedesco Goldschmied, entrambi indicanti primariamente l'orefice, descrivono anche, a seconda della lingua, diversi insetti (in svedese, norvegese e tedesco la coccinella, mentre in danese la libellula), i quali condividono con gli orefici la protezione e cura di materiali preziosi (Alinei \& Barros Ferreira, 1990). Il tedesco Schuhmacher «calzolaio» è decisamente più difficile da motivare, prima di tutto perché il lemma può far riferimento a diverse specie di insetto e non solo. Ciò vale anche per il sinonimo Schuster che, oltre a indicare la professione del calzolaio, indica anche un particolare tipo di ragno dalle zampe lunghe. Il termine antico tedesco sūtāri, deriva dal latino sutor «cucire» e quindi, in questo caso, si potrebbe far riferimento all'abilità del ragno di tessere ragnatele ${ }^{35}$. Dato che Schuster e Schuhmacher indicano la stessa professione, può darsi che la sinonimia si sia estesa non solo al significato primario ma successivamente anche a ragni e altri piccoli animali in grado di tessere. Da lì il significato del termine si è ulteriormente ampliato, andando a includere non solo gli insetti tessitori ma anche altre specie d'insetto.

\section{Popoli confinanti}

\begin{tabular}{|l|l|l|}
\hline Lemma & Traduzione letterale & Designatum \\
\hline Franzose (DE) & francese & scarafaggio, formica rossa \\
\hline Russe (DE) & russo & scarafaggio, formica rossa \\
\hline Schwabe (DE) & svevo & scarafaggio \\
\hline
\end{tabular}

Molti lemmi legati a popolazioni confinanti non particolarmente amate sono stati riutilizzati in chiave metaforica per descrivere diversi insetti ritenuti ripugnanti e infestanti. Ciò riguarda prevalentamente lo scarafaggio, che nella zona tedesca del Palatinato veniva chiamato Franzose «francese», Russe «russo» e Schwabe «svevo» ${ }^{36}$. L'uso denigratorio di questi termini veniva ulteriormente confermato dal fatto che non 
si limitavano a descrivere unicamente lo scarafaggio ma anche altri insetti considerati infestanti, come le formiche rosse. Questi popoli venivano considerati alla stregua dei cani e criticati aspramente per i loro modi, tanto che Russe poteva addirittura essere usato come nome di cane. Il termine per francese Franzose veniva usato per descrivere malattie sessualmente trasmissibili e la tubercolosi, oltre che a descrivere le formiche e una specifica razza suina. Anche in svedese infatti il termine fransoser era usato per riferirsi a diverse malattie sessualmente trasmissibili, inclusa la sifilide.

\section{Nomi familiari}

\begin{tabular}{|l|l|l|}
\hline Lemma & Traduzione letterale & Designatum \\
\hline Pfeifmutter (DE) & madre con la pipa & farfalle cavolaia \\
\hline Himmelsvaterlein (DE) & padrino del cielo & coccinella \\
\hline Schusterähnel (DE) & zietta del calzolaio & $\begin{array}{l}\text { coccinella, farfalle, libellula, ragno, } \\
\text { cervo volante, tarlo, cavalletta }\end{array}$ \\
\hline Sonnenähnel (DE) & zietta del sole & coccinella \\
\hline
\end{tabular}

Nelle società basate su caccia e raccolta, gli animali venivano spesso considerati come membri della famiglia. Venivano adorati ma allo stesso tempo cacciati e la vita intera del clan ruotava intorno a essi. Questo senso di familiarità era quindi motivato dal ruolo essenziale giocato da questi animali nella sussistenza del genere umano. L'area germanica regala diversi spunti interessanti: in Svezia l'orso veniva spesso chiamato «nonno» e la foca «fratello Lars». Ci sono anche diversi totem associati a questi animali che venivano adorati anche al di là del confine linguistico scandinavo (Alinei, 1984). Per quanto riguarda gli insetti, in tedesco abbiamo Himmelsvaterlein "piccolo padre del cielo» e diversi lemmi contenenti -ähnel tipo Schusterähnel «zietta del calzolaio» e Sonnenähnel «zietta del sole» (Alinei, 2002). Il primo si riferisce a diverse specie d'insetto come ad esempio la coccinella, la farfalla, la libellula, il ragno, il cervo volante, il tarlo o la cavalletta mentre il secondo indica prevalentemente la coccinella, vista come portatrice di bel tempo e legata al culto di Frau Sonne, divinità femminile del sole. Come precisato prima, Schuster veniva usato principalmente per indicare insetti tessitori e gli altri insetti, simili ma senza questa peculiarità, potevano esser quindi visti come dei parenti. Questo giustificherebbe l'uso di Schusterähnel. Il termine tedesco Pfeifmutter indicherebbe invece una specie di farfalla chiamata "cavolaia» anche se veniva usato più genericamente per tutti quelli insetti che attaccano e divorano piante con frutti a forma conica. La testa del composto potrebbe quindi essere interpretata come un possibile nome familiare «madre» o come una forma alternativa del tedesco Motte «falena» (Kemper, 1959).

\section{Streghe e megere}

\begin{tabular}{|l|l|l|}
\hline Lemma & Traduzione letterale & Designatum \\
\hline Smörgumma (SV) & megera del burro & coccinella \\
\hline
\end{tabular}




\begin{tabular}{|l|l|l|}
\hline Käringssjäl (SV) & anima della strega & farfalla \\
\hline Vevkjerring (NO,NN) & megera tessitrice & ragno \\
\hline Zauberweib (DE) & donna magica & coccinella \\
\hline Wasserhexe (DE) & strega delle acque & libellula \\
\hline
\end{tabular}

Le streghe rappresentano l'elemento di congiunzione tra l'umano e il soprannaturale. Nonostante siano delle figure umane, vengono generalmente associate al diavolo. Tra gli insetti, in particolar modo le farfalle, le libellule e, in misura minore, le coccinelle, venivano viste come manifestazioni della strega. Diversi lemmi aventi come referente la farfalla, e in parte anche la coccinella, hanno il burro o altri prodotti caseari come modificatori. Queste designazioni sono comuni in tutte le lingue germaniche: in alcune sono diventate le varianti standard mentre in altre vengono usate solo a livello dialettale. In alcuni lemmi come l'inglese butterfly «mosca o (ancora meglio) insetto volante del burro» il riferimento al burro è lampante mentre nel tedesco Schmetterling la motivazione lessicale è ormai divenuta opaca. Il lemma Schmetterling potrebbe derivare dall'antico sassone Schmetten «crema»" ${ }^{37}$. Simili sono anche altri nomi dialettali della farfalla quali Milchdieb e Molkendieb «ladro del latte», Butterlecker «leccaburro», Sahnelecker «leccacrema» e Buttervogel «creatura volante del burro» (Bächthold-Stäubli \& Hoffmann-Kreyer, 1986). Non mancano lemmi simili anche in svedese: ricordiamo smörgumma «vecchia del burro» e kärnanpiga «serva della zangola». La zangola, in svedese smörkärna, è uno strumento usato nella lavorazione del burro. Secondo la credenza popolare, le streghe potevano tramutarsi in farfalle e rubare burro, formaggio e latte, intrufolandosi nelle case ${ }^{38}$. Nel folclore svedese anche le coccinelle erano viste come delle streghe intente a rubare prodotti caseari (Pohl-Sennhauser, 2007). Altri nomi di insetto che si riferiscono a streghe e megere sono il norvegese vevkjerring «megera tessitrice ${ }^{39}$, usato per i ragni, e il tedesco Wasserhexe «strega dell'acqua» ${ }^{40} \mathrm{e}$ Hexenvogel «uccello o (ancora meglio) creatura volante della strega», usati in riferimento alla libellula, che si pensava fosse controllata dalle streghe e simboleggiasse il demonio.

\section{Creature sacre e magiche}

30 Non è sempre facile distinguere in quali casi l'insetto sia dotato di poteri magici propri e in quali gli vengono concessi da entità superiori. Gran parte di questi lemmi sono di fatto dei composti in cui il rapporto con la divinità o con la creatura magica di riferimento vengono espressi tramite il modificatore mentre la testa del composto, espressa obbligatoriamente in tutte le lingue germaniche, può riferirsi a oggetti o a creature terrene controllate da poteri superiori. Per esempio, nel termine svedese standard usato per indicare la libellula trollslända «fuso del troll», la libellula è descritta come un oggetto controllato dal troll o come un oggetto in cui il troll si può eventualmente trasformare. In considerazione della stratigrafia lessicale presentata da Alinei, il troll potrebbe essere classificato come una creatura antropomorfica pagana nonostante i tratti mostruoso-animali siano ancora largamente predominanti, non trattandosi quindi di una creatura antropomorfica a livello delle divinità del pantheon 
germanico. Di conseguenza, potrebbe essere considerato uno stadio intermedio tra lo zoomorfico e l'antropomorfico. Ciò indicherebbe che una chiara separazione tra questi due stadi non risulta essere poi così netta. Ma di fatto l'obbiettivo primario della stratificazione degli item lessicali è quello di fornire una linea guida ideale e non quello di dare una demarcazione netta dei diversi lemmi.

\section{La mara}

\begin{tabular}{|l|l|l|}
\hline Lemma & Traduzione letterale & Designatum \\
\hline Nachtmahr (DE) & mara notturna & falena \\
\hline mårå (SV) & Mara & libellula \\
\hline märrepil (SV) & freccia della mara & libellula \\
\hline märrekolv (SV) & freccia della mara & libellula \\
\hline
\end{tabular}

Oltre allo standard trollslända, in cui la libellula è interpretabile come strumento utilizzato dal troll, ci sono numerosi altri lemmi in cui la libellula viene descritta come un insetto prevalentemente negativo. Uno dei più interessanti è sicuramente la figura della mara, attestata in diversi termini dialettali svedesi mårå, märrepil e märrekolv ${ }^{41}$. Le mare sono delle entità femminili responsabili di causare incubi e sono presenti sia nel folklore germanico sia in quello slavo. In tedesco, il termine Mahr era usato principalmente nella Germania del nord mentre nel resto del paese è stato sostituito da un altro succubo chiamato Alp, presente nel composto Alptraum, termine ora usato per indicare l'incubo nel tedesco standard (Bächthold-Stäubli \& Hoffmann-Kreyer, 1986). Come raffigurato nell'olio su tela del pittore svizzero Johann Heinrich Füssli Mahre e Alpen potevano essere considerate due creature demoniache complementari. L'Alp era raffigurato come un coboldo accovacciato sul petto della vittima, di solito una donna, mentre la mara era raffigurata come una giumenta emaciata. Questa interpretazione della mara come cavallo potrebbe essere dovuta alla somiglianza tra il termine Mahr con Mähre, per l'appunto la giumenta. La somiglianza tra i due lemmi tedeschi può essere quindi alla base della fusione tra la mara e la giumenta nel folklore popolare: lo spirito malvagio si trasforma in cavallo e quindi il cavallo è visto come simbolo della creatura demoniaca. Come ulteriore conferma di questa ipotesi, numerosi nomi della libellula contengono il cavallo come testa del composto e il diavolo o una qualche creatura maligna, come il troll, nel modificatore: ad esempio il tedesco Teufelspferd «cavallo del diavolo» e il danese fandens ridehest «destriero del diavolo». Anche lo standard norvegese mareritt "cavalcata della mara» usato nello standard per indicare l'incubo sembra confermare questa teoria.

In tedesco dialettale il termine Nachtmahr può far riferimento non solo alla libellula ma anche alla falena che può quindi essere anch'essa considerata una manifestazione della mara. Nel folklore tedesco, le mare e le streghe potevano apparire anche sotto forma di falene ed erano ritenute responsabili di causare febbre, cattivi pensieri e incubi e di essere dei demoni della malattia (Pohl-Sennhauser, 2007). Anche l'inglese dragonfly può essere considerata una designazione pagana: la parola dragon è stata importata dal latino attraverso l'antico francese anche se originariamente si tratta di un termine 
greco antico drakon usato per indicare un serpente alato (Grimm, 1835). La motivazione lessicale di questa forma è, a sua volta, confermata da un'altra forma usata in inglese per descrivere la libellula: adderbolt, ossia «la freccia della vipera», anche qui un ulteriore riferimento a un rettile.

\section{L'orologio della morte}

\begin{tabular}{|l|l|l|}
\hline Lemma & Traduzione letterale & Designatum \\
\hline dødningur (NO, DK) & orologio della morte & tarlo \\
\hline Totenuhr (DE) & orologio della morte & tarlo \\
\hline
\end{tabular}

Nel norvegese e nel danese dialettale il tarlo è anche chiamato dødningur «orologio della morte» che, secondo i dizionari, potrebbe essere un prestito dal tedesco Totenuhr (Toten «morti»+ Uhr «orologio») $)^{42}$. Questo nomignolo che è stato dato alla specie Xestobium rufovillosium è riscontrabile anche nell'inglese death-watch beetle e sembra essere collegato al suono prodotto dal tarlo nel periodo dell'accoppiamento. Secondo delle antiche credenze, questo rumore ricorderebbe il ticchettio di un orologio che si fa sempre più vicino segnalando la morte della persona che lo ascolta ed è da interpretare quindi come un presagio nefasto (Bächthold-Stäubli \& Hoffmann-Kreyer, 1986). Tuttavia, questi lemmi potrebbero anche essere un caso di rianalisi e di etimologia popolare. Se consideriamo che la radice antico germanica -hnōr veniva usata per formare nomina agentis la motivazione lessicale dietro Totenuhr e dødningur cambierebbe drasticamente e acquisirebbe il significato di qualcuno (o qualcosa) portatore di morte (Tod «morte»+ *Nuhr).

\section{Spiriti di campo e demoni del grano}

A differenza dei casi presentati finora, qui possiamo notare alcuni composti che venivano associati, nella loro interezza, a un gruppo specifico di spiriti maligni, chiamati demoni del grano, quali «l'uomo dell'avena» e «la capra dell'avena», che potevano prendere la forma di insetti come ad esempio, la cavalletta. In effetti, la cavalletta era considerata un animale infestante e veniva accusata di distruggere $i$ campi; quindi non sorprende che venga associata ai demoni del grano e agli spiriti di campo. Probabilmente questi spiriti sono ancor più antichi delle divinità del pantheon germanico e potrebbero quindi essere classificati come appartenenti allo stadio zoomorfico nella teoria dei tre stadi teorizzata da Mario Alinei. Wilhlem Mannhardt ha descritti questi demoni in maniera esaustiva nella sua opera Die Korndämonen. Beitrag zur germanischen Sittenkunde (1868).

Secondo quanto riportato, nelle società agricole questi demoni erano inizialmente degli spiriti della fertilità che venivano venerati per ottenere un raccolto abbondante. Non avevano necessariamente forma umana e spesso assumevano forma animale, in special modo forma di lupi, maiali, scrofe, capre, pecore, orsi e persino gatti. Secondo la credenza popolare, le folate di vento erano branchi di scrofe o lupi che correvano liberamente tra i campi. Questi spiriti erano quindi demoni del vento e la loro presenza poteva essere percepita ogni qual volta si sentiva il vento soffiare. In alcune zone queste antiche credenze vennero poi cristianizzate: il gregge del signore vagava 
attraverso i campi di grano e Gesù era il loro pastore. Anche gli spiriti di campo finirono per prendere forma umana e la loro funzione è cambiata nel corso del tempo, diventando dei demoni la cui funzione era di tenere i bambini lontani dai campi di grano (Bächthold-Stäubli \& Hoffmann-Kreyer, 1986). Spiriti di campo quali il «caprone dell'avena» Haferbock e «capra dell'avena» Hafergeiß si sono poi tramutati nell'uomo dell'avena Hafermann, un uomo nero che doveva spaventare i bambini. Animali infestanti come le cavallette erano quindi la manifestazione tangibile di questi demoni e di conseguenza molte di queste designazioni vennero poi usate per indicare anche gli insetti.

\section{Sunna, Freya e la Vergine Maria}

\begin{tabular}{|l|l|l|l|}
\hline Lemma & Traduzione letterale & Designatum & Figura sacra \\
\hline Frauenkäferlein (DE) & $\begin{array}{l}\text { piccolo coleottero } \\
\text { della signora }\end{array}$ & coccinella & Sunna, Freya \\
\hline Sonnenkindchen (DE) & bambinello del sole & coccinella & Sunna \\
\hline Sonnenwürmchen (DE) & vermicello del sole & coccinella & Sunna \\
\hline $\begin{array}{l}\text { Mariehøne - Mariehøns } \\
\text { (NO, NN, DK) }\end{array}$ & gallo, pollo di Maria & $\begin{array}{l}\text { coccinella, } \\
\text { farfalle }\end{array}$ & Vergine Maria \\
\hline $\begin{array}{l}\text { (Jungfru Marie) } \\
\text { Nyckelpiga (SV) }\end{array}$ & serva della chiave & coccinella & Vergine Maria \\
\hline Marienkäfer (DE) & coleottero di Maria & coccinella & Vergine Maria \\
\hline Marienwürmchen (DE) & vermicello di Maria & coccinella & Vergine Maria \\
\hline Muttergotteskäfer (DE) & coleottero della madre di Dio & coccinella & Vergine Maria \\
\hline
\end{tabular}

Alcuni nomi della coccinella come Sonnenkindchen «bambinello del sole», Sonnenwürmchen «vermicello del sole», Sonnenkalb «vitello del sole», Sonnenkuh «mucca del sole» e Sonnenküken "pulcino del sole» sono spesso associati con alcune divinità pagane femminili e, di conseguenza, come risultato del sincretismo, con la Vergine Maria. Presumibilmente facevano riferimento a una dea chiamata Sol o Sunna, la personificazione del sole nel pantheon germanico (Lindow, 2001). Altri lemmi come il tedesco dialettale Frauenkäferlein «piccolo coleottero della signora», Frauachüeli «mucca della signora» e l'inglese ladybird, ladybug e ladycow contengono una generica figura femminile come modificatore. Questi lemmi, attribuiti successivamente alla vergine Maria, erano molto probabilmente usati inizialmente per altre dee pagane. Nell'antico nordico la coccinella era chiamata freyjuhöna "gallina di Freya» prima di essere cristianizzato in marihøne "gallina di Maria» nell'odierno norvegese (Grimm, 1835). Ciò detto, anche lemmi tedeschi contenenti il modificatore Frauen-, come Frauenkäferlein e Frauachüeli, apparentemente generici, potrebbero in realtà nascondere un legame con Freya, dea della fertiltà. L'antico nordico Freya era in origine un termine generico e ha poi sviluppato un'accezione nuova riferendosi alla figura femminile per antonomasia, 
ovvero una dea. E' possibile che lo stesso sia avvenuto in inglese e che, di conseguenza, anche i lemmi inglesi con il modificatore lady abbiano una connessione con il culto di Freya. Lo standard svedese nyckelpiga (serva della chiave) presenta una motivazione lessicale cristiana e la coccinella è qui consacrata alla vergine Maria. Tuttavia, questa interpretazione sembra aver sostituito una versione pagana preesistente legata al culto delle dee del pantheon nordico Freya e Frigg. Secondo la leggenda, Fulla, servitrice di Freya, aveva il compito di sorvegliare il portagioielli della sua signora. Nyckelpiga, ossia la serva della chiave, potrebbe di fatto riferisi alla versione cristianizzata di Fulla, custode della chiave del paradiso per volontà di Maria. Questa chiave esisterebbe sulla terra con il nome di primula veris, chiamata in svedese gullviva o anche Jungfru Marie nyckel «chiave della Vergine Maria». La coccinella sarebbe quindi la custode di un fiore in grado di aprire le porte del paradiso. Un altro nome svedese della primula veris è nyckelblomma o nyckelblomster «fiore chiave», usato anche per indicare un altro fiore, l'orchidea ${ }^{43}$. Quest'ultima è conosciuta in islandese con il nome friggjargras «erba di Frigg» (Hendriks, 2017). Altre specie di orchidea si riferiscono invece a dei santi, lo svedese Johannesnycklar «chiavi di Giovanni», Johannes nyckelblomster «fiore della chiave di Giovanni» e il norvegese Johannesnøklar «chiavi di Giovanni» si riferiscono all'orchis militaris mente Sankt Pers nycklar «chiavi di san Pietro» designa l'orchis maculata ${ }^{44}$. Anche in tedesco abbiamo diversi nomi per la primula veris: Schlüsselblume «fiore della chiave», Himmelschlüssel «chiavi del cielo» e Sankt Peters Schlüssel «chiavi di San Pietro» ${ }^{45}$. Secondo la leggenda, una svista di San Pietro avrebbe portato alla nascita di questo fiore: un giorno gli caddero infatti le chiavi del paradiso, che finirono sulla terra tramutandosi in un fiore, la primula veris (Eckehardt, 2008). La coccinella era quindi spesso invocata per portare al richiedente le chiavi del paradiso sotto forma di fiore, insieme ad altri doni, tra cui anche dei figli, come mostrato nella seguente rima infantile svizzera (Storl, 2014):

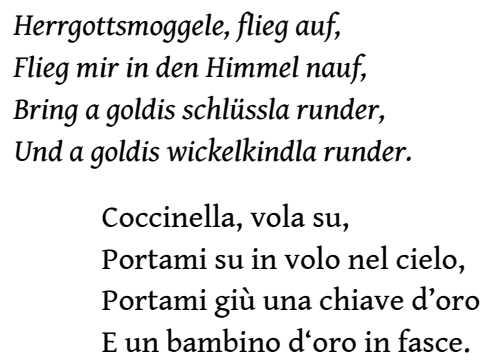

Altri nomi della coccinella con la Vergine Maria come modificatore del composto sono Marienkäfer «coleottero di Maria», il dialettale Muttergotteskäfer «coleottero della madre di Dio» e Marienwürmchen «vermicello di Maria».

\section{San Giovanni}

\begin{tabular}{|l|l|l|}
\hline Lemma & Traduzione & Designatum \\
\hline Johanniskäfer (DE) & coleottero di San Giovanni & lucciola, coccinella \\
\hline Johannisvögelchen (DE) & uccellino di San Giovanni & lucciola \\
\hline Johannisfliege (DE) & insetto volante di San Giovanni & lucciola \\
\hline
\end{tabular}




\begin{tabular}{|l|l|l|}
\hline Johanniswurm (DE) & verme di San Giovanni & lucciola \\
\hline
\end{tabular}
modo per indicare la lucciola. Questi lemmi sono però per lo più legati al periodo di comparsa dell'insetto e raramente fanno riferimento all'effettivo culto del santo. La lucciola appare infatti solitamente all'inizio dell'estate e il termine Johanniskäfer «coleottero di Giovanni» è dovuto al fatto che questi insetti compaiano solitamente verso fine giugno e San Giovanni è, per l'appunto, celebrato nella notte tra il 23 e il 24 giugno, solo un paio di giorni dopo il solstizio d'estate. Infatti, un altro nome della lucciola è Sonnenwendkäfer, ovvero "coleottero del solstizio». Nella notte di San Giovanni, viene solitamente acceso un fuoco (tedesco Johannisfeuer «fuoco di Giovanni») e, secondo la leggenda, sarebbe San Giovanni stesso ad accenderlo.

San Giovanni, come simbolo dell'inizio dell'estate, compare anche in altri composti come nel tedesco Johannisbeere «bacca di Giovanni», ovvero il mirtillo, che matura a giugno o in Johanniskraut «erba di Giovanni» ${ }^{46}$ e Johannisblut «sangue di Giovanni» o ancora nello svedese Johannesört «erba di Giovanni», tutti nomi indicanti l'Hypericum perforatum, che fiorisce proprio in questo periodo. San Giovanni è celebrato anche in Scandinavia: in Svezia è una vera e propria festa, conosciuta come midsommarafton «notte di mezza estate». Gli elementi della luce e del fuoco sono ancora più rappresentativi in questo caso, dato che si è soliti accendere un falò. Questo rituale è probabilmente antecedente al cristianesimo anche se scarseggiano dati a riguardo ${ }^{47}$.

\section{Dio, Gesù e il Paradiso}

\begin{tabular}{|l|l|l|l|}
\hline Lemma & Traduzione & Designatum & Figura sacra \\
\hline Herrgottskäfer (DE) & coleottero di Dio & coccinella & Dio \\
\hline Gottestierchen (DE) & animaletto di Dio & coccinella & Dio \\
\hline Liebgottrosslein (DE) & ronzino del Buon Dio & coccinella & Dio \\
\hline Vorherreshane (DE) & gallo del Signore & coccinella & Dio \\
\hline Jessvallpika (SV) & pastorella di Gesù & coccinella & Gesù \\
\hline Himmelswurm (DE) & verme del cielo & coccinella & Paradiso \\
\hline Himmelschäfchen (DE) & pecorella del cielo & coccinella & Paradiso \\
\hline
\end{tabular}

Anche Dio viene spesso usato come modificatore dei composti, in particolar modo nei nomi della coccinella. Ecco alcuni esempi: il tedesco dialettale Liebgottsrosslein «piccolo destriero del buon Dio», Vorherreshane "gallo del Signore», Gottestierchen «animaletto di Dio» e Herrgottskäfer «coleottero di Dio». In alcune definizioni si ricorre persino al cielo come modificatore. Sempre in tedesco abbiamo Himmelschäfchen "pecorella del cielo», Himmelswurm «verme del cielo» e Himmelsvaterlein «piccolo padre del cielo». Il tedesco, come d'altronde l'inglese, ricorre ancora più spesso delle lingue scandinave a termini 
cristiani, visto che nella zona mitteleuropea l'influsso del cristianesimo è stato ben più rilevante. Queste definizioni fanno ovviamente parte del primo stadio della teoria dei tre stadi elaborata da Mario Alinei, che può, a sua volta, essere scisso in due sottocategorie: uno strato cattolico più antico seguito da uno protestante. Secondo questa teoria, i nomi contenenti un riferimento alla Vergine Maria apparterrebbero allo stadio cattolico e sarebbero stati poi sostituiti da altri modificatori, come Dio e Gesù, con l'avvento del protestantesimo. Ciò implicherebbe che le divinità femminili germaniche e scandinave vennero inizialmente sostituite da Maria, a cui poi subentrò Dio nella fase protestante ${ }^{48}$.

\section{Concludendo}

41 Il fatto che esistano nomi di insetto simili in aree molto distanti come il Norrland svedese e la Baviera tedesca conferma l'esistenza di un antico culto comune, o per lo meno, di alcuni tratti culturali condivisi tra le diverse aree germaniche. In realtà, questa visione condivisa del mondo, e nella fattispecie del mondo degli insetti, va ben al di là del confine linguistico germanico, tanto che definizioni simili si trovano anche in molte altre lingue indoeuropee e non solo. Possiamo sicuramente notare una tendenza universale a descrivere gli insetti ricorrendo ai mezzi linguistici e culturali a disposizione del parlante. Nei nomi ritenuti più antichi gli insetti sono dotati di poteri magici propri e spesso vengono invocati con delle richieste ben specifiche da parte del richiedente. Con l'avvento di una società stratificata a livello sociale, le divinità hanno acquisito la forma di chi deteneva il potere, ovvero l'uomo. Perciò, molti poteri inizialmente associati a divinità zoomorfe sono diventati tipici di dei antropomorfi e, con il cristianesimo, gli dèi pagani sono a loro volta stati sostituiti da santi, dalla Vergine Maria o persino da Dio stesso. Gli insetti però sono riusciti a mantenere, almeno in parte, il loro potere magico spesso però in veste di messaggeri di un'entità superiore, fungendo così da punto di unione tra l'umano e il sovrannaturale. Quindi, non è più la natura, e di conseguenza le divinità zoomorfe tra cui gli insetti, a controllare l'uomo ma è l'uomo che si è convinto sempre più di poter controllare la natura. Ciò traspare ovviamente anche dal modo in cui il mondo animale viene interpretato e descritto.

\section{BIBLIOGRAFIA}

Aline Mario, 1984, L'evoluzione dal totemismo al cristianesimo popolare studiate negli sviluppi semantici dei dialetti italiani, in Dal totemismo al cristianesimo popolare, sviluppi semantici nei dialetti italiani ed europei, Alessandria, Edizioni dell'Orso.

ALINEI Mario, 1984, L'evoluzione dal totemismo al cristianesimo popolare studiate negli sviluppi semantici dei dialetti italiani, «Quaderni di Semantica», vol. IV, p. 3-29. 
ALINEI Mario \& BARRoS FERREIRA Manuela, 1990, Coccinelle, in Atlas Linguarum Europae, vol. I-4, Assen / Maastricht, Van Gorcum, carte 42-44, commentaires p. 99-199.

ALINEI Mario, 1997, Magico-Religious Motivations in European Dialects: A Contribution to Archaeolinguistics, «Dialectologia et Geolinguistica», n 5, p. 3-30.

ALINEI Mario, 2002, Nomi di animali, animali come nomi: cosa ci insegnano i dialetti sul rapporto fra esseri umani ed animali, Trento, Iprase.

BARROS FERREIRA Manuela, 1997, Ver Luisant, in ALE, vol. I-5, Roma, IPZS.

BÄCHTHOLD-STÄUBLI Hanns \& HOFFMANN-KREYER Eduard, 1986, Handwörterbuch des deutschen Aberglaubens, Berlin, De Gruyter.

ECKEHARDT J. Jäger, 2008, Exkursionsflora von Deutschland: Krautige Zier- und Nutzpflanzen, vol. 5, Berlin / Heidelberg, Spektrum Akademischer Verlag.

Esopo, 2016, Favole, Milano, Rizzoli.

GRIMM Jacob, 2012, Deutsche Mythologie [1835], Nabu Press.

HENDRIKS Cor, 2017, The Folklore of the Ladybird, capitolo 1. Disponibile online all'indirizzo <https:// robscholtemuseum.nl/wp-content/uploads/2017/05/Cor-Hendriks-The-Folklore-of-theLadybird-1.pdf>.

KEMPER Heinrich, 1959, Die tierischen Schädlinge im Sprachgebrauch, Berlin, Duncker \& Humblot.

LinDow John, 2001, Norse Mythology: A Guide to the Gods, Heroes, Rituals and Beliefs, New York, Oxford University Press.

MANNHARDT Wilhelm, 2018, Die Korndämonen: Beitrag zur germanischen Sittenkunde [1868], Forgotten books.

NiLSSON Anders, 2008, Dialektala namn på insekter och andra småkryp i Norrland, «Natur i norr», vol. $27, n^{\circ} 1$, p. 1-12.

POE Edgar Allan, 2016, Complete Tales and Poems of Edgar Allan Poe, Sterling.

POHL-SENNHAUSER Ida, 2007, Rattenschwanz und Schneckenschleim: Aberglaube oder Vergessene Volksmedizin?, Wien / Köln / Weimar, Böhlau Verlag.

STORL Wolf Dieter, 2014, Die alte Göttin und ihre Pflanzen: Wie wir durch Märchen zu unserer Urspiritualität finden, Berlin, Kailash Auflage.

\section{Sitografia}

Den Danske Ordbog: <https://ordnet.dk/ddo>.

Den virtuella floran: <http://linnaeus.nrm.se/flora/mono/orchida/orchi/orchmil.html>.

Deutsches Wörterbuch von Jacob Grimm und Wilhelm Grimm: <www.dwds.de/d/wb-1dwb>.

Duden: <www.duden.de>.

Frühneuhochdeutsches Wörterbuch: <www.fwb-online.de>.

Meyers Großes Konversationslexikon: <http://woerterbuchnetz.de/cgi-bin/WBNetz/[...]>.

Pfälzisches Wörterbuch: <http://woerterbuchnetz.de/cgi-bin/WBNetz/[...]>. 
Rheinisches Wörterbuch: <http://dwv.uni-trier.de/de/die-woerterbuecher/das-rheinischewoerterbuch>.

Svenska Akademiens Ordbok: <https://svenska.se/saob>.

Svensk Etymologisk Ordbok: <http://runeberg.org/svetym/0425.html>.

Schweizerisches Idiotikon: <www.idiotikon.ch>.

Wörterbuch der deutschen Gegenwartssprache: <www.dwds.de/wb>.

Wörterbuch der elsässischen Mundarten: <http://woerterbuchnetz.de/cgi-bin/WBNetz/

wbgui_py?sigle=ElsWB>.

\section{NOTE}

1. Nelle tabelle di riferimento si ricorre a delle abbreviazioni relative alle lingue prese in considerazione: DE (tedesco); DK (danese); NO (norvegese); SV (svedese).

2. Svenska Akademiens Ordbok, s.v. blindsticka.

3. Deutsches Wörterbuch von Jacob Grimm und Wilhelm Grimm, s.v. Teufelsnadel.

4. Deutsches Wörterbuch von Jacob Grimm und Wilhelm Grimm, s.v. Gottestierchen.

5. Rheinisches Wörterbuch, s.v. Sommertierchen.

6. Bokmålsordboka, s.v. sommerfugl. Den Danske Ordbog, s.v. sommerfugl. Svenska Akademiens Ordbok, s.v. sommarfågel.

7. Deutsches Wörterbuch von Jacob und Wilhelm Grimm, s.v. Maivogel. Rheinisches Wörterbuch, s.v. Maivogel.

8. Deutsches Wörterbuch von Jacob und Wilhelm Grimm, s.v. Sommervöglein.

9. Wörterbuch der elsässischen Mundarten, s.v. Hexenvogel.

10. Pfälzisches Wörterbuch, s.v. Johannisvogel.

11. Deutsches Wörterbuch von Jacob und Wilhelm Grimm, s.v. Buttervogel.

12. Den Danske Ordbog, s.v. marihøne. Bokmålsordboka, s.v. marihøns.

13. Deutsches Wörterbuch von Jacob und Wilhelm Grimm, s.v. Springhahn.

14. Deutsches Wörterbuch von Jacob und Wilhelm Grimm, s.v. Teufelspferd.

15. Svenska Akademiens Ordbok, s.v. geting.

16. Den Danske Ordbog, s.v. gedehams.

17. Duden, s.v. Hornisse.

18. Pfälzisches Wörterbuch, s.v. Rebbock. Rheinisches Wörterbuch, s.v. Heubock.

19. Svenska Akademiens Ordbok, s.v. torbagge.

20. Pfälzisches Wörterbuch, s.v. Himmelsschäfchen.

21. Duden, s.v. Motschekiebchen.

22. Den Danske Ordbog, s.v. eghjort.

23. Pfälzisches Wörterbuch, s.v. Hirschmann.

24. Bokmålsordboka, s.v. bille. Nynorskordboka, s.v. bille.

25. Duden, s.v. Käfer.

26. Pfälzisches Wörterbuch, s.v. Muttergotteskäfer. Pfälzisches Wörterbuch, s.v. Herrgottskäfer.

27. Frühneuhochdeutsches Wörterbuch, s.v. Johanniskäfer.

28. Meyers Großes Konversationslexikon, s.v. Klopfkäfer.

29. Frühneuhochdeutsches Wörterbuch, s.v. Johannisfliege.

30. Duden, s.v. Wurm.

31. Den Danske Ordbog, s.v. orm. Den Danske Ordbok, s.v. slange. Svenska Akademiens Ordbok, s.v. mask. Svenska Akademiens Ordbok, s.v. mott. 
32. Schweizerisches Idiotikon, s.v. Wasserjungfrau.

33. Den Danske Ordbog, s.v. vandnymf. Svenska Akademiens Ordbok, s.v. flickslända. Svenska Akademiens Ordbok, s.v. jungfruslända. Det Norske Akademins Ordbok, s.v. vannymfe. Den Danske Ordbog, s.v. vandnymf.

34. Det Norske Akademins Ordbok, s.v. gullkonge. Svenska Akademiens Ordbok, s.v. guldbrud.

35. Duden, s.v. Schuster.

36. Pfälzisches Wörterbuch, s.v. Russe. Pfälzisches Wörterbuch, s.v. Schwabe.

37. Duden, s.v. Schmetterling.

38. Svenska Akademiens Ordbok, s.v. smörkärna.

39. Det Norske Akademis Ordbok, s.v. vevkjerring.

40. Deutsches Wörterbuch von Jacob und Wilhelm Grimm, s.v. Wasserhexe.

41. Svensk Etymologisk Ordbok.

42. Den Danske Ordbog, s.v. dødningur.

43. Svenska Akademiens Ordbok, s.v.jungfru.

44. Den virtuella floran.

45. Deutsches Wörterbuch von Jacob und Wilhelm Grimm, s.v. Schlüsselblume.

46. Wörterbuch der deutschen Gegenwartssprache: s.v. Johanniskäfer, Johannisfeuer, Johannisbeere, Johanniskraut.

47. Svenska Akademiens Ordbok, s.v. Johannes, midsommarafton.

48. Schweizerisches Idiotikon.

\section{RIASSUNTI}

L'obbiettivo del mio articolo è di classificare i nomi degli insetti in tedesco e nelle lingue scandinave, individuando e descrivendo le categorie motivazionali più ricorrenti. I lemmi analizzati sono stati quindi suddivisi in quattro principali macrocategorie, a seconda della diversa motivazione lessicale soggiacente: 1) «gli oggetti»; 2) «gli animali», a loro volta divisi in «animali di grande taglia» (pollame, ovini, bovini, suini, ecc.) e «insetti», spesso iperonimi o termini generici quali la mosca, usata per indicare qualsiasi insetto volante (il termine nelle lingue germaniche deriva infatti dal verbo «volare») o il verme, usato per gran parte degli insetti striscianti. 3) «Gli esseri umani», categoria che include sia designazioni più generiche sia riferimenti a popoli, professioni e nomi familiari. L'ultima categoria è composta da 4) «creature magiche e/o sacre» e raggruppa in sé figure sia pagane sia cristiane. Molte di queste ultime nascono poi proprio come entità pagane, successivamente reinterpretate in chiave cristiana per opera del sincretismo religioso. A metà tra la terza e la quarta categoria, potremmo poi inserire la categoria degli «esseri umani con poteri magici», quale ad esempio la strega, che funge da anello di congiunzione tra la categoria dell'umano e quella del magico/sacro.

L'objectif de mon article est de classer les noms d'insectes en allemand et en scandinave, en identifiant et en décrivant les catégories de motivation les plus fréquentes. Les lemmes analysés ont ensuite été subdivisés en quatre macro-catégories principales, en fonction de la motivation lexicale sous-jacente : 1) «objets»;2) 《animaux », eux-mêmes divisés en «grands animaux » (volailles, moutons, bovins, porcins, etc.) et « insectes », souvent des hyperonymes ou des termes génériques tels que mouche, utilisés pour désigner tout insecte volant (le terme en langues germaniques dérive du verbe "voler») ou «ver», utilisé pour désigner la plupart des insectes 
rampants ; 3) «êtres humains ", une catégorie qui comprend à la fois des désignations plus génériques et des références aux peuples, aux professions et aux noms de famille; 4) « créatures magiques et/ou sacrées» qui regroupe des figures païennes et chrétiennes. Beaucoup de ces dernières sont alors neés comme des entités païennes, puis réinterprétées avec une clé chrétienne par le biais du syncrétisme religieux. À mi-chemin entre les troisième et quatrième catégories, nous pourrions ensuite insérer les « êtres humains dotés de pouvoirs magiques ", tels que la sorcière, qui agit comme un lien entre la catégorie de l'humain et celle de la magie/sacré.

The aim of my article is to classify insect names in German and Scandinavian languages, identifying and describing the most recurring lexical categories. The lexical items have thus been divided in four main macro-categories, according to the underlying lexical motivation: 1) "objects"; 2) "animals", in their turn divided in "large-sized animals" (poultry, ovines, bovines and swines) and "insects", often hypernyms or generic terms, like "fly" used to indicate any flying insect (the terms stems from the verb "to fly") or "worm", used for a great number of crawling insects. 3) The category "human beings" includes generic designations or more specific references to peoples, professions and kinship names. 4) The last category "magic and/or sacred creatures" includes both heathen and Christian figures. Many of the Christian designations were originally heathen entities, which were later reinterpreted and Christianised due to religious syncretism. An additional category "human beings with magic powers", including "the witch", could be placed halfway between the third and the fourth category, and serves as a link between the human and magic/sacred world.

\section{INDICE}

Mots-clés : noms d'insectes, motivation lexicale, lexicologie, etholinguistique, noms composés Parole chiave : nomi di insetti, motivazione lessicale, lessicologia, etnolinguistica, nomi composti

Keywords : insect names, lexical motivation, lexicology, ethnolinguistics, compound names

\section{AUTORE}

\section{CLAUDIO BARTOLESCHI}

Università degli Studi di Milano

claudio.bartoleschi@gmail.com 